\title{
The Debate Over Alcohol Consumption: How Much Is Too Much?
}

PAMELA HALLQUIST VIALE, RN, MS, CNS, ANP

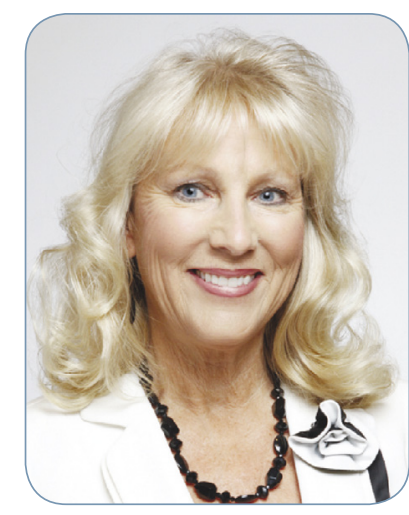

J Adv Pract Oncol 2019;10(1):18-19 https://doi.org/10.6004/jadpro.2019.10.1.1 (c) 2019 Harborside ${ }^{\mathrm{TM}}$ tudies regarding safe levels of alcohol consumption are relatively easy to find in the literature. However, the conclusions of the studies vary, and it's easy to get confused over the most accurate recommendations regarding the consumption of alcohol. In fact, it's difficult to truly understand where alcohol falls into place regarding health and risks/benefits. And recent data have emerged to eclipse our current beliefs regarding safe alcohol consumption. What are we supposed to believe is the truth?

\section{PREVIOUS RESEARCH}

In 2017, researchers published a paper in the Journal of the American College of Cardiology touting the benefits of alcohol and concluding that light-tomoderate drinking can lower the risk of mortality from all causes, including cardiovascular disease (Xi et al., 2017). The study included data from 333,247 participants surveyed from 1997 to 2009. Although the study showed that high alcohol consumption is linked to a variety of health issues, the authors stated that alcohol in moderation is widely recommended, and the study demonstrated that light-to-moderate drinking might have protective effects against cardiovascular disease, a major cause of death in both sexes. In fact, an accompanying editorial to the paper stated that for most older persons, the overall benefits of light drinking clearly outweighed the possible cancer risk associated with the habit. The authors also noted that the small risk of breast, colorectal, or oral cancer is relatively small and should be considered in the context of each individual risk (de Gaetano \& Costanzo, 2017).

\section{THE LANCET STUDY ON ALCOHOL USE AND RISK}

The most recent study to report on alcohol use and health risk was published in The Lancet by the Global Burden of Disease (GBD) 2016 Alcohol Collaborators. An accompanying paper describes this new study as the most comprehensive estimate of the global burden of alcohol use to date, using methodologic improvements of previous iterations to produce the data (Burton \& Sheron, 2018). Estimates of alcohol use, attributed deaths, and disability-adjusted life years (DALYs) were calculated for 195 locations from 1990 through 2016, including for both sexes and for ages 15 through 95 and older (GBD 2016 Alcohol Collaborators, 2018). The study used 694 data sources of individual and population-level con- 
sumption and 592 prospective and retrospective studies on the risk of alcohol use.

Alcohol use was the seventh leading risk factor for both deaths and DALYs in 2016, producing $2.2 \%$ of age-standardized female deaths and $6.8 \%$ of age-standardized male deaths; for ages 15 to 49 , alcohol use was the leading risk factor, with $3.8 \%$ of female deaths and $12.2 \%$ of male deaths attributable to alcohol use globally. Although the three leading causes of attributable deaths in this age group were tuberculosis, road injuries, and selfharm, for populations aged 50 and older, cancers accounted for a large number of total alcohol-attributable deaths in 2016. The authors of this large study concluded that alcohol use is a leading risk factor for disease burden globally, accounting for almost $10 \%$ of deaths among populations aged 15 to 49 . The study demonstrated that the safest level of drinking is none, conflicting with most health guidelines that report up to two drinks per day produces health benefits (GBD 2016 Alcohol Collaborators, 2018).

\section{RESEARCH AND}

\section{CONFOUNDING RESULTS}

How do we get such different results from published studies? We know that experimental studies are usually blinded and randomized, factors that strengthen the results obtained from the study. Observational studies are harder to control, as the researchers cannot control the study volunteers completely. Statistical rigor usually calls for large studies, which strengthens the studies' power. The study reported in The Lancet is global and included data from 195 locations as well as a thorough review of many previous studies and data sources. The study covered a significant period of time, which also strengthens the results. Are there possible explanations for doubting the conclusions?

\section{EVALUATING THE RESEARCH}

Because the study called for the elimination of all drinking as the safest level for global health, the news outlets picked up the story and reported the results. Some of the reports quoted experts such as a Stanford researcher who believed that the study, although good at estimating the disease burden of alcohol use, should have focused on the dangers of excessive drinking instead of calling for no alcohol use at all (Belluz, 2018). Belluz also noted that the researcher stated that nutritional science is dependent on observational studies, which may weaken the studies' results. Advanced practitioners questioning patients regarding their alcohol use know the difficulties of getting an honest calculation of actual use. Therefore, we may assume that the results demonstrate an association but not a causal result (Belluz, 2018).

\section{ADVANCED PRACTITIONER'S ROLE}

So, what should we conclude from the latest research study on the benefits and risks of drinking alcohol? One can probably safely assume that drinking alcohol has risks for many, and that drinking more alcohol increases risk. But can we assume that no alcohol is the safest route for people to follow? Until we get a randomized trial with a large population of participants, we may not truly know the answer. Advanced practitioners should know the latest literature and counsel patients appropriately on risk, specifically for cancer and cardiovascular disease, as well as on unsafe behavior. We should continue to rigorously evaluate published research and implement findings as necessary in our patient population. Stay tuned: The definitive recommendations on safe alcohol use and risk may be coming soon. And in the meantime, The Lancet study has produced some powerful data on the global risks of alcohol consumption.

\section{References}

Belluz, J. (2018). Is one drink per day really unsafe? That new alcohol study, explained. Retrieved from https://www. vox.com/science-and-health/2018/8/29/17790118/alcohol-lancet-health-study

Burton, R., \& Sheron, N. (2018). No level of alcohol consumption improves health. Lancet. Advance online publication. https://doi.org/10.1016/S0140-6736(18)31571-X

de Gaetano, G. \& Costanzo, S. (2017). Alcohol and health: Praise of the $\mathrm{J}$ curves. Journal of the American College of Cardiology, 70(8), 923-925. http://doi.org/10.1016/j.jacc.2017.07.710

GBD 2016 Alcohol Collaborators. (2018). Alcohol use and burden for 195 countries and territories, 1990-2016: A systematic analysis for the Global Burden of Disease Study 2016. Lancet. Advance online publication. https:// doi.org/10.1016/S0140-6736(18)31310-2

Xi, B., Veeranki, S. P., Zhao, M., Ma, C., Yan, Y., \& Mi, J. (2017). Relationship of alcohol consumption to all-cause, cardiovascular, and cancer-related mortality in U.S. adults. Journal of the American College of Cardiology, 70(8), 913922. https://doi.org/10.1016/j.jacc.2017.06.054 